\title{
A CRITERION FOR THE EXISTENCE OF BIHARMONIC GREEN'S FUNCTIONS
}

\author{
LEO SARIO
}

(Received 1 August 1974)

\begin{abstract}
The biharmonic Green's function of a simply supported plate is generalized to Riemannian manifolds and shown to exist if and only if the harmonic measure of the ideal boundary is square integrable.

The harmonic Green's function was originally introduced as the electrostatic potential of a point charge in a grounded system. Its characterization by the fundamental singularity and vanishing boundary values permitted its generalization to regular subregions $\Omega$ of an abstract Riemann surface or Riemannian manifold $R$. The Green's function $g$ on $R$ was then defined as the directed limit, if it exists, of the Green's function $g_{\Omega}$ on $\Omega$ as $\{\Omega\}$ exhausts $R$. The distinction of Riemann surfaces and Riemannian manifolds into hyperbolic and parabolic types according as $g$ does or does not exist is still a cornerstone of the harmonic classification theory.

The biharmonic Green's function $\gamma$ also has an important physical meaning: it is the deflection of a thin elastic plate under a point load. However, in sharp contrast with the harmonic case, nothing seems to be known about its existence on noncompact spaces. The purpose of the present paper is to initiate research on this fundamental problem of biharmonic classification theory.

Biharmonicity being not meaningful on abstract Riemann surfaces, our aim is to generalize the definition of the biharmonic Green's function to Riemannian manifolds $R$ and to explore its existence on them. On a regular subregion $\Omega$ of $R$, there exist two biharmonic Green's functions, to be denoted by $\beta$ and $\gamma$, with a biharmonic fundamental singularity, and with boundary data $\beta=\partial \beta / \partial n=0$ and $\gamma=\Delta \gamma=0$. For dimension 2 , both functions give the deflection under a point load of a thin plate which is clamped or simply supported at the edges,
\end{abstract}

Sponsored by the U. S. Army Research Office, Grant DA-AROD-31-124-73-G39, University of California, Los Angeles. 
respectively. Our present investigation deals exclusively with $\gamma$. The corresponding function $\gamma_{\Omega}$ on $\Omega$ increases with $\Omega$, and we set $\gamma=\lim _{\Omega \rightarrow R} \gamma_{\Omega}$ on $R$.

We first study the existence of $\gamma$ on the Euclidean $N$-space $R^{N}$. The result is fascinatingly simple: $\gamma$ exists if and only if $N>4$. By way of preparation we recall the peculiar behavior of the biharmonic fundamental singularity at the origin: $r^{2} \log r$ for $N=2, r$ for $N=3, \log r$ for $N=4$, and $r^{4-N}$ for $N>4$.

No parabolic Riemannian manifold carries $\gamma$. For a hyperbolic Riemannian manifold $R$ we deduce a natural criterion: If $\bar{R}_{0} \subset \Omega \subset R$, and $\omega_{\Omega}$ is harmonic on $\Omega-\bar{R}_{0}$ with boundary values 1 on $\partial R_{0}, 0$ on $\partial \Omega$, and we denote by $\omega=\lim _{\Omega \rightarrow R} \omega_{\Omega}$ the harmonic measure of $\partial R_{0}$ on $R-R_{0}$, then $\gamma$ exists on $R$ if and only if $\omega \in L^{2}\left(R-R_{0}\right)$. An essential step of the reasoning is the proof of the independence of the existence of $\gamma$ on the choice of the fundamental singularity. This property allows us to introduce the class $O_{\Gamma}$ of Riemannian manifolds which do not carry $\gamma$, in analogy with the class $O_{G}$ of parabolic manifolds.

As a simple illustration of our criterion we generalize the above result that $R^{N} \in O_{\mathrm{r}}$ if and only if $N \leqq 4$. We ask whether one could induce $\gamma$ to exist even for these low dimensions by replacing the Euclidean metric $d s=|d x|$ by $d s=\left(1+r^{2}\right)^{\alpha}|d x|$, with the constant $\alpha$ sufficiently large. The answer is intriguing: the resulting space is in $O_{r}$ for $N \leqq 4$ regardless of what $\alpha$ is chosen. For $N>4, \gamma$ continues to exist if and only if $\alpha>-\frac{1}{2}$.

The usefulness of the criterion $\omega \in L^{2}$ for $R \notin O_{r}$ lies in the fact that it also applies if there is no way of obtaining an expression for the approximating Green's functions $\gamma_{\Omega}$, and even if nothing is known about the metric of an arbitrarily small neighborhood of the ideal boundary of the Riemannian manifold.

Applications of our criterion to relations between $O_{\mathrm{r}}$ and other biharmonic null classes will be discussed in later studies [see Ralston and Sario (to appear), Sario (to appear), Wang (1974), Wang (to appear), in the attached Bibliography of recent work in the field].

Throughout this paper, $\Delta$ will stand for the Laplace-Beltrami operator $d \delta+\delta d$, biharmonicity being defined by $\Delta^{2} \cdot=0$. Let $\Omega$ be a regular subregion of a Riemannian manifold $R$, carrying the biharmonic Green's function $\gamma_{\Omega}(x, y)$ on $\bar{\Omega}$, with the biharmonic fundamental singularity at $y \in \Omega$ and with the boundary data

$$
\gamma_{\Omega}\left|\partial \Omega=0, \quad \Delta \gamma_{\Omega}\right| \partial \Omega=0 .
$$

In terms of the harmonic Green's function $g_{\Omega}(x, y)$ on $\Omega$ with singularity $y$, the function $\gamma_{\Omega}(x, y)$ has the well known integral representation

$$
\gamma_{\Omega}(x, y)=\int_{\Omega} g_{\Omega}(x, z) g_{\Omega}(z, y) d z,
$$


with $d z$ the Riemannian volume element at $z$. To see this, it suffices to verify

$$
\Delta \int_{\Omega} g_{\Omega}(x, z) g_{\Omega}(z, y) d z=g_{\Omega}(x, y),
$$

where $\Delta$ is taken with respect to $x$. For every $\phi \in C_{0}^{\infty}$,

$$
\begin{gathered}
\int_{\Omega} \Delta\left(\int_{\Omega} g_{\Omega}(x, z) g_{\Omega}(z, y) d z\right) \cdot \phi(x) d x=\int_{\Omega}\left(\int_{\Omega} g_{\Omega}(x, z) g_{\Omega}(z, y) d z\right) \Delta \phi(x) d x \\
=\int_{\Omega}\left(\int_{\Omega} g_{\Omega}(x, z) \Delta \phi(x) d x\right) g_{\Omega}(z, y) d z=\int_{\Omega} g_{\Omega}(z, y) \phi(z) d z .
\end{gathered}
$$

Therefore (3) is true in the sense of distributions and a fortiori in the conventional sense.

We introduce the biharmonic Green's function $\gamma(x, y)$ on a Riemannian manifold $R$ by setting

$$
\gamma(x, y)=\lim _{\Omega \rightarrow R} \gamma_{\Omega}(x, y)
$$

provided the limit exists for some exhaustion $\{\Omega\}$. We shall show in No. 4 that the existence is independent of the exhaustion $\{\Omega\}$ and the choice of the singularity y.

We start by examining the existence of $\gamma(x, y)$ in an illuminating special case, the Euclidean $N$-space $R^{N}$. Here the computation is elementary. A function $h(r)$ is harmonic if $\Delta h(r)=0$, i.e., $-r^{-N+1}\left(r^{N-1} h^{\prime}(r)\right)^{\prime}=0$. We obtain

$$
h(r)= \begin{cases}a \log r+b, & N=2, \\ a r^{-N+2}+b, & N>2,\end{cases}
$$

where $a, b$ are arbitrary constants. If $u(r)$ belongs to the class $H^{2}$ of nonharmonic biharmonic functions on $R^{N}$, then $\Delta u(r)=h(r)$. A straightforward integration yields the biharmonic, quasiharmonic, harmonic, and constant components of $u(r)$ :

$$
u(r)= \begin{cases}a r^{2} \log r+b r^{2}+c \log r+d, & N=2, \\ a r+b r^{2}+c r^{-1}+d, & N=3, \\ a \log r+b r^{2}+c r^{-2}+d, & N=4, \\ a r^{-N+4}+b r^{2}+c r^{-N+2}+d, & N>4,\end{cases}
$$

with $a, b, c, d$ arbitrary constants. 
Let $B_{\rho}$ be a ball $\{r<\rho\}$ and take $x \in B_{\rho},|x|=r$. In view of (6) and $\Delta r^{2}=-2 N$, the biharmonic Green's function on $B_{\rho}$ with singularity 0 is

$$
\gamma_{\rho}(x, 0)= \begin{cases}r^{2} \log \frac{r}{\rho}-\left(r^{2}-\rho^{2}\right), & N=2, \\ -r+\rho+\frac{1}{3}\left(r^{2}-\rho^{2}\right) \rho^{-1}, & N=3, \\ -\log \frac{r}{\rho}+\frac{1}{4}\left(r^{2}-\rho^{2}\right) \rho^{-2}, & N=4, \\ r^{-N+4}-\rho^{-N+4}+\frac{N-4}{N}\left(r^{2}-\rho^{2}\right) \rho^{-N+2}, & N>4,\end{cases}
$$

where the signs have been so chosen that $\gamma_{\rho}>0$. As $\rho \rightarrow \infty,\left\{B_{\rho}\right\}$ exhausts $R^{N}$, and we obtain

$$
\gamma(x, 0)=r^{-N+4}, \quad N>4,
$$

whereas $\lim _{\rho \rightarrow \infty} \gamma_{\rho}=\infty$ for $N=2,3,4$.

We have proved:

THEOREM 1. The biharmonic Green's function exists on the Euclidean $N$-space if and only if $N>4$.

3

For further illustration we recall the simple computation showing the significance of the biharmonic Green's function in expressing values of a biharmonic function $u$ in terms of the boundary values of $u$ and $\Delta u$. Given a regular subregion $\Omega$ of a Riemannian manifold, let $y \in \Omega, u \in H^{2}(\bar{\Omega})$, and $\gamma \in H^{2}(\Omega-\{y\}) \cap C^{3}(\bar{\Omega}-\{y\})$. Take a compact hypersurface $\alpha$ enclosing $y$ and suppose that as $\alpha$ shrinks to $y$,

$$
\int_{\alpha} \cdot \Delta \gamma * d u \rightarrow 0, \int_{\alpha} \cdot \Delta u * d \gamma \rightarrow 0, \int_{\alpha} \gamma * d \Delta u \rightarrow 0
$$

Then

$$
u(y)=\frac{1}{F(\Delta \gamma)} \int_{\partial \Omega} u * d \Delta \gamma-\Delta \gamma * d u+\Delta u * d \gamma-\gamma * d \Delta u
$$

with $F(\Delta \gamma)=\int_{\alpha} * d \Delta \gamma$ the flux of $\Delta \gamma$. In fact, by Stokes' formula the integral on the right taken along $\partial \Omega-\alpha$ is

$$
\begin{gathered}
(d u, d \Delta \gamma)-\left(u, \Delta^{2} \gamma\right)-(d \Delta \gamma, d u)+(\Delta \gamma, \Delta u) \\
+(d \Delta u, d \gamma)-(\Delta u, \Delta \gamma)-(d \gamma, d \Delta u)+\left(\gamma, \Delta^{2} u\right)=0
\end{gathered}
$$


where the inner products are taken over the region bounded by $\partial \Omega \cup \alpha$. If $\langle\varepsilon\rangle$ stands for a quantity which $\rightarrow 0$ as $\alpha \rightarrow y$, then

$$
\int_{\alpha} u * d \Delta \gamma=\int_{\alpha}(u(y)+\langle\varepsilon\rangle) * d \Delta \gamma \rightarrow u(y) F(\Delta \gamma),
$$

and (10) follows.

In the Euclidean case of No. $2, \Omega$ is the ball $\{r<\rho\}, y$ the origin, and $\gamma=\gamma_{\rho}$. Accordingly,

$$
u(0)=\frac{1}{F(\Delta \gamma)} \int_{r-\rho} u * d \Delta \gamma_{\rho}+\Delta u * d \gamma_{\rho}
$$

provided (9) holds.

Denote by $d S$ the surface element on the sphere $S_{\rho}=\{r=\rho\}$ and let $\alpha$ be the sphere $\{r=\delta<\rho\}$. For $N=2$, (7) gives

$$
\begin{aligned}
& * d \gamma_{\rho}=\left(2 r \log \frac{r}{\rho}-r\right) d S, \Delta \gamma_{\rho}=-4 \log \frac{r}{\rho}, \\
& * d \Delta \gamma_{\rho}=-4 r^{-1} d S, F\left(\Delta \gamma_{\rho}\right)=-4 \int_{\alpha} r^{-1} d S=-\int_{0}^{2 \pi} 4 d \phi=-8 \pi .
\end{aligned}
$$

On the other hand, $|u|,|\partial u / \partial n|,|\Delta u|$ are bounded on $B_{\rho}$, and as $\delta \rightarrow 0$,

$$
\begin{aligned}
& \left|\int_{\alpha} \cdot \Delta \gamma_{\rho} * d u\right|<M\left|\delta \log \frac{\delta}{\rho}\right| \rightarrow 0, \\
& \left|\int_{\alpha} \Delta u * d \gamma_{\rho}\right|<M\left|2 \delta \log \frac{\delta}{\rho}-\delta\right| \delta \rightarrow 0, \\
& \left|\int_{\alpha} \gamma_{\rho} * d \Delta u\right|=c(\delta) F(\Delta u)=0 .
\end{aligned}
$$

Equation (11) follows, with $F\left(\Delta \gamma_{\rho}\right)=-8 \pi$.

For $N=3$, (7) gives

$$
\begin{aligned}
* d \gamma_{\rho} & =\left(-1+\frac{2}{3} r \rho^{-1}\right) d S, \Delta \gamma_{\rho}=2 r^{-1}-2 \rho^{-1}, \\
* d \Delta \gamma_{\rho} & =-2 r^{-2} d S, F\left(\Delta \gamma_{\rho}\right)=-2 \int r^{-2} r^{2} d \omega=-8 \pi,
\end{aligned}
$$

where $d \omega$ is the area element of the unit sphere. As $\delta \rightarrow 0$,

$$
\begin{aligned}
& \left|\int_{\alpha} \Delta \gamma_{\rho} * d u\right|<M\left(2 \delta^{-1}-2 \rho^{-1}\right) \cdot 4 \pi \delta^{2} \rightarrow 0, \\
& \left|\int_{\alpha} \Delta u * d \gamma_{\rho}\right|<M\left|-1+\frac{2}{3} \delta \rho^{-1}\right| \cdot 4 \pi \delta^{2} \rightarrow 0, \\
& \left|\int_{\alpha} \gamma_{\rho} * d \Delta u\right|=c(\delta) F(\Delta u)=0,
\end{aligned}
$$

and (11) follows, with $F\left(\Delta \gamma_{\rho}\right)=-8 \pi$. 
For $N=4$, (7) gives

$$
\begin{aligned}
* d \gamma_{\rho} & =\left(-r^{-1}+\frac{1}{2} r \rho^{-2}\right) d S, \Delta \gamma_{\rho}=2 r^{-2}-2 \rho^{-2}, \\
* d \Delta \gamma_{\rho} & =-4 r^{-3} d S, F\left(\Delta \gamma_{\rho}\right)=-4 \int r^{-3} r^{3} d \omega=-4 \omega_{3},
\end{aligned}
$$

where $\omega_{3}$ is the area of the unit 3-sphere. As $\delta \rightarrow 0$,

$$
\begin{aligned}
& \left|\int_{\alpha} \Delta \gamma_{\rho} * d u\right|<M\left(2 \delta^{-2}-2 \rho^{-2}\right) \omega_{3} \delta^{3} \rightarrow 0, \\
& \left|\int_{\alpha} \Delta u * d \gamma_{\rho}\right|<M\left|-\delta^{-1}+\frac{1}{2} \delta \rho^{-2}\right| \omega_{3} \delta^{3} \rightarrow 0, \\
& \left|\int_{\alpha} \gamma_{\rho} * d \Delta u\right|=c(\delta) F(\Delta u)=0
\end{aligned}
$$

and (11) follows, with $F\left(\Delta \gamma_{\rho}\right)=-4 \omega_{3}$.

Finally, for $N>4,(7)$ gives

$$
\begin{aligned}
* d \gamma_{\rho}= & (N-4) r^{-N+3}\left(-1+\frac{2}{N}\left(\frac{r}{\rho}\right)^{N-2}\right) d S, \\
\Delta \gamma_{\rho}= & (N-4)\left[-(N-3)\left(r^{-N+2}+\frac{2}{N(N-3)} \rho^{-N+2}\right)\right. \\
& \left.-(N-1)\left(-r^{-N+2}+\frac{2}{N} \rho^{-N+2}\right)\right], \\
* d \Delta \gamma_{\rho}= & -2(N-2)(N-4) r^{-N+1} d S, \\
F\left(\Delta \gamma_{\rho}\right)= & -2(N-2)(N-4) \omega_{N-1},
\end{aligned}
$$

where $\omega_{N-1}$ is the area of the unit $(N-1)$-sphere. As $\delta \rightarrow 0$,

$$
\begin{aligned}
& \left|\int_{\alpha} \Delta \gamma_{\rho} * d u\right|<M\left|c_{1} \delta^{-N+2}+c_{2}\right| c_{3} \delta^{N-1} \rightarrow 0, \\
& \left|\int_{\alpha} \Delta u * d \gamma_{\rho}\right|<M\left|c_{1} \delta^{-N+3}+c_{2} \delta\right| c_{3} \delta^{N-1} \rightarrow 0, \\
& \left|\int_{\alpha} \gamma_{\rho} * d \Delta u\right|=c(\delta) F(\Delta u)=0,
\end{aligned}
$$

and (11) follows, with $F\left(\Delta \gamma_{\rho}\right)=-2(N-2)(N-4) \omega_{N-1}$.

In summary, (11) is true for all $N$. For our present purpose of illustrating the use of $\gamma$ this will suffice. Equation (11) generalizes, however, in various directions. First, since the fundamental singularity is locally defined, the above reasoning continues to hold when $u(0)$ is replaced by $u(y)$ with $y \in B_{\rho}$, and $\gamma_{\rho}(x, 0)$ by $\gamma_{\rho}(x, y)$. On a Riemannian manifold $R$, the geodesic distance $d$ is, in a 
sufficiently small neighborhood of $y$, a constant multiple of the Euclidean $r$ in a parametric ball, added by a function which together with its derivatives is bounded. Therefore, the above elementary reasoning, mutatis mutandis, remains valid and (11) holds for $u(y)$ when $S_{\rho}$ is replaced by the boundary $\partial \Omega$ of a regular subregion of $R$, and $\gamma_{\rho}$ by the biharmonic Green's function $\gamma_{\Omega}(x, y)$ on $\Omega$.

We return to our existence problem. The proof of the following intermediate result is essential.

Proposition. The existence of a biharmonic Green's function $\gamma$ on $a$ Riemannian manifold is independent of the exhaustion and the choice of the singularity.

Proof. Let $\{\Omega\}$ be some exhaustion of $R$ as in No. 1. By (2) and Lebesgue's Dominated Convergence Theorem, if a biharmonic Green's function $\gamma$ on $R$ with singularity $y$ exists, it can be written as

$$
\gamma(x, y)=\int_{R} g(x, z) g(z, y) d z
$$

where $g$ is the harmonic Green's function on $R$, and the integral is the directed limit of $\int_{\Omega} g_{\Omega}(x, z) g_{\Omega}(z, y) d z$ as $\Omega \rightarrow R$. Thus we have immediately the independence of the exhaustion. It remains to show that if $\gamma$ exists for some $\left(x_{1}, y_{1}\right)$, then it exists for any $\left(x_{2}, y_{2}\right)$.

From (12), we see that $\gamma$ can exist only on hyperbolic manifolds, and

$$
\int_{R} g\left(x_{1}, z\right) g\left(z, y_{1}\right) d z<\infty .
$$

Choose a regular subregion $R_{0}$ of $R$ containing $x_{1}, x_{2}, y_{1}$, and $y_{2}$, with boundary $\alpha$. Clearly (13) is equivalent to

$$
\int_{R-R_{0}} g\left(x_{1}, z\right) g\left(z, y_{1}\right) d z<\infty,
$$

which in turn is equivalent to

$$
\int_{R-R_{0}} g\left(x_{1}, z\right) g\left(y_{1}, z\right) d z<\infty,
$$

by virtue of the symmetry of the harmonic Green's function. Since $g(x, z)$, $g(y, z)>0$ on $R_{0}$ for each $z \in R-\bar{R}_{0}$, Harnack's inequality gives

$$
g(y, z)<K g(x, z)
$$


for all $x, y$ in $R_{0}$, with the constant $K$ depending only on $R$ and $R_{0}$. Therefore,

$$
K^{-1} \int_{R-R_{0}} g(x, z)^{2} d z<\int_{R-R_{0}} g(x, z) g(y, z) d z<K \int_{R-R_{0}} g(x, z)^{2} d z,
$$

and we conclude that $\gamma\left(x_{1}, y_{1}\right)$ exists if and only if $\int_{R-R_{0}} g\left(x_{1}, z\right)^{2} d z<\infty$, that is,

$$
g\left(\cdot, x_{1}\right) \in L^{2}\left(R-R_{0}\right) .
$$

Next we shall show that $g\left(\cdot, x_{1}\right) \in L^{2}\left(R-R_{0}\right)$ if and only if the harmonic measure $\omega$ of $\alpha$ on $R-R_{0}$ belongs to $L^{2}\left(R-R_{0}\right)$. Recall that $\omega$ is the limit of the harmonic function $\omega_{\Omega}$ on $\Omega-\bar{R}_{0}$ with boundary values 1 on $\alpha, 0$ on $\partial \Omega$, for some and hence every exhaustion $\{\Omega\}$ of $R$ with $\bar{R}_{0} \subset \Omega$. The function $\omega$ always exists, and $\omega \equiv 1$ if and only if $R$ is parabolic. Set

$$
m_{\Omega}=\min _{\alpha} g_{\Omega}\left(\cdot, x_{1}\right), \quad M_{\Omega}=\max _{\alpha} g_{\Omega}\left(\cdot, x_{1}\right) .
$$

By the maximum principle for harmonic functions,

$$
m_{\Omega} \omega_{\Omega} \leqq g_{\Omega}\left(\cdot,, x_{1}\right) \leqq M_{\Omega} \omega_{\Omega}
$$

on $\bar{\Omega}-R_{0}$. On letting $\Omega \rightarrow R$, we obtain

$$
m \omega \leqq g\left(\cdot, x_{1}\right) \leqq M \omega
$$

on $R-R_{0}$, with

$$
m=\min _{\alpha} g\left(\cdot, x_{1}\right), \quad M=\max _{\alpha} g\left(\cdot, x_{1}\right) .
$$

Thus $g\left(\cdot, x_{1}\right) \in L^{2}\left(R-R_{0}\right)$ if and only if $\omega \in L^{2}\left(R-R_{0}\right)$. Since $g\left(\cdot, x_{2}\right) \in$ $L^{2}\left(R-R_{0}\right)$ is characterized by the same condition, we have proved the existence of $\gamma\left(x_{2}, y_{2}\right)$ as a consequence of that of $\gamma\left(x_{1}, y_{1}\right)$.

We also conclude from the above reasoning that $\lim _{\Omega \rightarrow R} \gamma_{\Omega}$ is either identically infinite or a biharmonic function $\gamma$ on $R-\{y\}$ with the fundamental singularity $y$.

In view of the Proposition, we may introduce the class of Riemannian manifolds

$$
O_{\Gamma}=\{R \mid \gamma \text { does not exist }\} .
$$

Let $\tilde{O}_{\Gamma}$ be the class of manifolds which do carry $\gamma$. From the proof of the Proposition, we have the following criterion:

THEOREM 2. Every parabolic Riemannian manifold $R$ belongs to $O_{\mathrm{r}}$. $A$ hyperbolic $R$ belongs to $\tilde{O}_{\Gamma}$ if and only if $\omega \in L^{2}\left(R-R_{0}\right)$ for some and hence every $R_{0}$. 
Using Theorem 2, we can extend Theorem 1. We ask whether $\gamma$ exists even for the low dimensions if $R^{N}$ is generalized to the Riemannian manifold $R_{\alpha}^{N}$ with the same base manifold $\{r<\infty\}$ but with the metric

$$
d s=\left(1+r^{2}\right)^{\alpha}|d x|,
$$

$\alpha$ a constant. For a harmonic $h(r)$ we have by direct computation

$$
h(r)=a \int_{1}^{r} r^{-N+1}\left(1+r^{2}\right)^{-(N-2) a} d r+b
$$

as $r \rightarrow \infty$. If $\alpha \leqq-\frac{1}{2}$, this is unbounded, hence $R_{\alpha}^{N} \in O_{G} \subset O_{r}$. For $\alpha>-\frac{1}{2}$, the harmonic measure $\omega$ satisfies

$$
\omega(r) \sim a r^{-(N-2)(2 \alpha+1)},
$$

and the $L^{2}$-norm of $\omega(r)$ over the annulus $(1, r)$ is

$$
\|\omega\|_{2}^{2} \sim c \int_{1}^{r} r^{-2(N-2)(2 \alpha+1)+N-1+2 N \alpha} d r=c \int_{1}^{r} r^{-2(N-4) \alpha-(N-3)} d r .
$$

This is bounded if and only if $N>4$ and $\alpha>-\frac{1}{2}$. We have proved that $R_{\alpha}^{N}$ fails to carry $\gamma$ for $N \leqq 4$ regardless of how rapidly or slowly the metric $\left(1+r^{2}\right)^{\alpha}|d x|$ grows:

THEOREM 3. $R_{\alpha}^{N} \in \tilde{O}_{\Gamma}$ if and only if $N>4$ and $\alpha>-\frac{1}{2}$.

The author is indebted to Professor Cecilia Wang for a careful checking of the manuscript.

\section{Bibliography}

L. Chung (to appear), 'Manifolds carrying bounded quasiharmonic but no bounded harmonic functions', Math. Scand.

L. Chung (to appear), 'Asymptotic behavior and biharmonic degeneracy'.

L. Chung and L. Sario (to appear), 'Harmonic and quasiharmonic degeneracy of Riemannian manifolds', Tôhoku Math. J.

L. Chung, L. Sario and C. Wang (1973), 'Riemannian manifolds with bounded Dirichlet finite polyharmonic functions', Ann. Scuola Norm. Sup. Pisa 27, 1-6.

L. Chung, L. Sario and C. Wang (to appear), 'Quasiharmonic $L^{p}$ functions on Riemannian manifolds', Ann. Scuols Norm. Sup. Pisa.

D. Hada, L. Sario and C. Wang.(1975), 'Dirichlet finite biharmonic functions on the Poincaré $N$-ball', J. Reine Angew. Math. 272, 92-101.

D. Hada, L. Sario and C. Wang (1974), ' $N$-manifolds carrying bounded but no Dirichlet finite harmonic functions', Nagoya Math. J. 54, 1-6.

D. Hada, L. Sario and C. Wang (to appear), 'Bounded biharmonic functions on the Poincaré $\mathbf{N}$-ball', Kōdai Math. Sem. Rep. 
O. Haupt (1913), 'Über das asymptotische Verhalten der Lösungen gewisser linearer gewöhnlicher Differentialgleichungen', Math. Z. 48, 289-292.

E. Hille (1952), 'Behavior of solutions of linear second order differential equations', Ark. Mat. 2, 25-41.

Y. K. Kwon, L. Sario and B. Walsh (1971), 'Behavior of biharmonic functions on Wiener's and Royden's compactifications', Ann. Inst. Fourier (Grenoble) 21, 217-226.

N. Mirsky, L. Sario and C. Wang (1973), 'Bounded polyharmonic functions and the dimension of the manifold', J. Math. Kyoto Univ. 13, 529-535.

N. Mirsky, L. Sario and C. Wang (1974), 'Parabolicity and existence of bounded or Dirichlet finite polyharmonic functions', Rend. Ist. Mat. Univ. Trieste 6, 1-9.

M. Nakai and L. Sario (1967), 'Completeness and function-theoretic degeneracy of Riemannian spaces', Proc. Nat. Acad. Sci. 57, 29-31.

M. Nakai and L: Sario (1971), 'Biharmonic classification of Riemannian manifolds', Bull. Amer. Math. Soc. 77, 432-436.

M. Nakai and L. Sario (1972), 'Quasiharmonic classification of Riemannian manifolds', Proc. Amer. Math. Soc. 31, 165-169.

M. Nakai and L: Sario (1971), 'Dirichlet finite biharmonic functions with Dirichlet finite Laplacians', Math. Z. 122, 203-216.

M. Nakai and L. Sario (1971), 'A property of biharmonic functions with Dirichlet finite Laplacians', Math. Scand. 29, 307-316.

M. Nakai and L. Sario (1973), 'Existence of Dirichlet finite biharmonic functions', Ann. Acad. Sci. Fenn. A: I. 532, 1-33.

M. Nakai and L. Sario (1973), 'Existence of bounded biharmonic functions', J. Reine Angew. Math. 259, 147-156.

M. Nakai and L. Sario (1972), 'Existence of bounded Dirichlet finite biharmonic functions', Ann. Acad. Sci. Fenn. A: I. 505, 1-12.

M. Nakai and L. Sario (1972), 'Biharmonic functions on Riemannian manifolds', Continuum Mechanics and Related Problems of Analysis (Nauka, Moskow, 1972).

J. Ralston and L. Sario (to appear), 'A relation between biharmonic Green's functions of simply supported and clamped bodies'.

L. Sario (1968-1970), 'Biharmonic and quasiharmonic functions on Riemannian manifolds', Duplicated lecture notes (University of California, Los Angeles, 1968-1970).

L. Sario (1974), 'Quasiharmonic degeneracy of Riemannian N-manifolds', Ködai Math. Sem. Rep. 26, 53-57.

L. Sario (1974), 'Completeness and existence of bounded biharmonic functions on a Riemannian manifold', Ann. Inst. Fourier (Grenoble) 24, 311-317.

L. Sario (1974), 'Biharmonic measure', Ann. Acad. Sci. Fenn. A. I. 587, 1-18.

L. Sario (to appear), 'Biharmonic Green's functions and harmonic degeneracy', J. Math. Kyoto Univ.

L. Sario and M. Nakai (1970), Classification Theory of Riemann Surfaces (Springer Verlag, 1970).

L. Sario and C. Wang (1972), 'The class of ( $p, q)$-biharmonic functions', Pacific J. Math. 41, 799-808.

L. Sario and C. Wang (1974), 'Counterexamples in the biharmonic classification of Riemannian 2-manifolds', Pacific J. Math. 50, 159-162.

L. Sario and C. Wang (1972), 'Generators of the space of bounded biharmonic functions', Math. $Z$. 127, 273-280.

L. Sario and C. Wang (1973), 'Quasiharmonic functions on the Poincaré N-ball', Rend Mat. (4) 6, 1-14.

L. Sario and C. Wang (1974), 'Riemannian manifolds of dimension $N \geqq 4$ without bounded biharmonic functions'. J. London Math. Soc. (2) 7, 635-644. 
L. Sario and C. Wang (1973), 'Existence of Dirichlet finite biharmonic functions on the Poincaré 3-ball', Pacific J. Math. 48, 267-274.

L. Sario and C. Wang (1974), 'Negative quasiharmonic functions', Tôhoku Math. J. 26, 85-93.

L. Sario and C. Wang (1973), 'Radial quasiharmonic functions', Pacific J. Math. 46, 515-522.

L. Sario and C. Wang (1972), 'Parabolicity and existence of bounded biharmonic functions', Comm. Math. Helv. 47, 341-347.

L. Sario and C. Wang (1973), 'Positive harmonic functions and biharmonic degeneracy', Bull Amer. Math. Soc. 79, 182-187.

L. Sario and C. Wang (1974), 'Parabolicity and existence of Dirichlet finite biharmonic functions', $J$. London Math.'Soc. 8, 145-148.

L. Sario and C. Wang (1973), 'Harmonic and biharmonic degeneracy', Kōdai Math. Sem. Rep. 25, 392-396.

L. Sario ạd C. Wang (1975), 'Harmonic $L^{p}$-functions on Riemannian manifolds', Kōdai Math. Sem. Rep. 26, 204-209.

L. Sario, C. Wang and M. Range (1971), Biharmonic projection and decomposition', Ann. Acad. Sci. Fenn. A.' I. 494, 1-14.

C. Wang (1974), 'Biharmonic Green's function and quasiharmonic degeneracy', Math. Scand. 35, $38-42$.

C. Wang (to appear), 'Biharmonic Green's function and biharmonic degeneracy', Math. Scand.

C. Wang and L. Sario (1972), 'Polyharmonic classification of Riemannian manifolds', J. Math. Kyoto Uhiv. 12, 129-140.

\section{Georgina Avenue}

\section{Santa Monica}

California, 90402

U.S.A. 